\title{
Evidence for Solid Planets from Kepler's Near-Resonance Systems
}

\author{
Man Hoi Lee ${ }^{1}$, D. Fabrycky ${ }^{2,3}$ and D. N. C. Lin $^{3}$ \\ ${ }^{1}$ Dept. of Earth Sciences and Dept. of Physics, The University of Hong Kong, Hong Kong \\ ${ }^{2}$ Dept. of Astronomy \& Astrophysics, University of Chicago, \\ 5640 S. Ellis Ave., Chicago, IL 60637, USA \\ ${ }^{3}$ UCO/Lick Observatory, University of California, Santa Cruz, CA 95064, USA
}

\begin{abstract}
The multiple-planet systems discovered by the Kepler mission show an excess of planet pairs with period ratios just wide of exact commensurability for first-order resonances like $2: 1$ and $3: 2$. In principle, these planet pairs could be in resonance if their orbital eccentricities are sufficiently small, because the width of first-order resonances diverges in the limit of vanishingly small eccentricity. We consider a widely-held scenario in which pairs of planets were captured into first-order resonances by migration due to planet-disk interactions, and subsequently became detached from the resonances, due to tidal dissipation in the planets. In the context of this scenario, we find a constraint on the ratio of the planet's tidal dissipation function and Love number that implies that some of the Kepler planets are likely solid. However, tides are not strong enough to move many of the planet pairs to the observed separations, suggesting that additional processes are at play.
\end{abstract}

Keywords. celestial mechanics, solar system: formation, planetary systems: formation

\section{Introduction}

The first 16 months of observations by the Kepler space telescope have led to the discovery of 361 multiple-transit candidate systems (Fabrycky et al. 2012), and almost all of them are real multiple-planet systems (Lissauer et al. 2012). Although most of the planet pairs are not in or near mean-motion resonances, there is an excess of planet pairs with orbital period ratios just wide of the first-order 2:1 and 3:2 resonances and a deficit of pairs just narrow of the resonances (Lissauer et al. 2011; Fabrycky et al. 2012). The excess and deficit occur within a few percent of exact commensurabilities. It is useful to separate the sample into two groups: one with the radius of the inner planet $R_{1}<2 R_{\oplus}$ (Earths and super-Earths) and another with $R_{1}>2 R_{\oplus}$ (Neptunes and above). Both pairs with $R_{1}<2 R_{\oplus}$, and pairs with $R_{1}>2 R_{\oplus}$ show an excess just outside $3: 2$ and a deficit just inside $2: 1$, but there is not an obvious excess just outside $2: 1$ for pairs with $R_{1}<2 R_{\oplus}$ (although the statistics are noisy due to small numbers).

\section{Resonant Planet Pairs}

For first-order, $j:(j-1)$, mean-motion resonances like $2: 1$ and $3: 2$, there are two eccentricity-type resonance angles,

$$
\theta_{1}=(j-1) \lambda_{1}-j \lambda_{2}+\varpi_{1} \quad \text { and } \quad \theta_{2}=(j-1) \lambda_{1}-j \lambda_{2}+\varpi_{2},
$$

where $\lambda_{i}$ is the mean longitude of planet $i$ and $\varpi_{i}$ is the longitude of periapse $(i=1$ and 2 for the inner and outer planets, respectively). At least one of these angles must librate about a fixed value for the pair to be in resonance, if we ignore inclination resonances. 
The resonance induced periapse precession is usually retrograde, i.e., $\dot{\varpi}_{i}<0$. In the example of the 2:1 resonance in GJ 876 , both angles librate about $0^{\circ}$, and the periapses are observed to precess at an average rate of $\dot{\varpi}_{i}=-41^{\circ} \mathrm{yr}^{-1}$ (Laughlin \& Chambers 2001; Rivera \& Lissauer 2001; Lee \& Peale 2002; Laughlin et al. 2005). If the resonance angle $\theta_{i}$ is librating,

$$
\dot{\theta}_{i}=(j-1) n_{1}-j n_{2}+\dot{\varpi}_{i}=0
$$

on average, or

$$
\frac{P_{2}}{P_{1}}-\frac{j}{(j-1)}=-\frac{\dot{\varpi}_{i}}{(j-1) n_{2}}>0,
$$

where $n_{i}$ is the mean motion, $P_{i}=2 \pi / n_{i}$ is the orbital period, and $\dot{\varpi}_{i}<0$ due to the resonance. So $P_{2} / P_{1}$ should in fact be slightly larger than the exact period ratio for a resonant pair.

In the vicinity of a $j:(j-1)$ resonance, if we assume coplanar orbits, the Hamiltonian to the lowest order in the orbital eccentricities $e_{i}$ gives

$$
\dot{\varpi}_{1}=\alpha n_{1}\left(M_{2} / M_{*}\right) C_{1} / e_{1} \quad \text { and } \quad \dot{\varpi}_{2}=-n_{2}\left(M_{1} / M_{*}\right) C_{2} / e_{2}
$$

where $M_{*}$ is the stellar mass, $M_{i}$ is the planetary mass, $\alpha=a_{1} / a_{2}$, and $a_{i}$ is the orbital semimajor axis (e.g., Lee 2004). For 2:1, the coefficients $C_{1}=-1.190$ and $C_{2}=0.428$. For 3:2, $C_{1}=-2.025$ and $C_{2}=2.444$. Thus $\dot{\varpi}_{i} \propto-1 / e_{i}$ and $P_{2} / P_{1}-j /(j-1)$ could be large and positive, if the eccentricities are small. This is different from higher-order resonances. For example, for a second-order resonance, $\dot{\varpi}_{1}$ involves terms that are either independent of the eccentricities or proportional to $e_{2} / e_{1}$ (similarly, $e_{1} / e_{2}$ for $\dot{\varpi}_{2}$ ).

Convergent migration of planets due to interactions with the protoplanetary disk can result in capture into mean-motion resonances (e.g., Bryden et al. 2000; Kley 2000). This is the most likely scenario for the origin of the 2:1 resonance in the GJ 876 system (Lee \& Peale 2002). If the growth of eccentricity due to continued migration within the resonance is balanced by the damping of eccentricity by planet-disk interactions, the eccentricities would reach equilibrium values determined by the ratio of the rates of eccentricity damping and migration. A natural question arises as to whether the Kepler near-resonance pairs are simply resonance pairs with very small eccentricities (and hence large positive departure of $P_{2} / P_{1}$ from exact commensurability) due to large eccentricity damping during disk-induced migration. Most of the Kepler candidate planets are sufficiently small that they are unable to open gaps in the protoplanetary disks and should undergo type I migration. For classic type I migration, the migration and eccentricity damping timescales are (Ward 1997; Tanaka et al. 2002; Artymowicz 1993)

$$
\frac{a}{\dot{a}}=\frac{1}{C_{a}} \frac{M_{*}}{M_{p}} \frac{M_{*}}{\Sigma a^{2}}\left(\frac{H}{a}\right)^{2} \frac{P}{2 \pi} \quad \text { and } \quad \frac{e}{\dot{e}}=\frac{1}{9 C_{e}} \frac{M_{*}}{M_{p}} \frac{M_{*}}{\Sigma a^{2}}\left(\frac{H}{a}\right)^{4} \frac{P}{2 \pi},
$$

respectively, where $C_{a} \approx 3, C_{e} \approx 0.1, M_{p}$ is the planetary mass, $\Sigma$ is the surface mass density of the disk, and $H / a$ is the dimensionless scale height of the disk. The ratio

$$
K_{e}=\left|\frac{\dot{e} / e}{\dot{a} / a}\right|=\frac{9 C_{e}}{C_{a}}\left(\frac{H}{a}\right)^{-2} .
$$

For $H / a=0.05$ and $0.1, K_{e}=120$ and 30 , respectively.

We have performed direct numerical orbit integrations using the symplectic integrator SyMBA modified to include forced migration and eccentricity damping (Lee \& Peale 2002; Lee 2004). Figure 1 shows a convergent migration calculation with $M_{*}=1 M_{\odot}$ and $M_{1}=$ $M_{2}=10 M_{\oplus}$. The planets are initially far from the 2:1 mean-motion commensurability, 

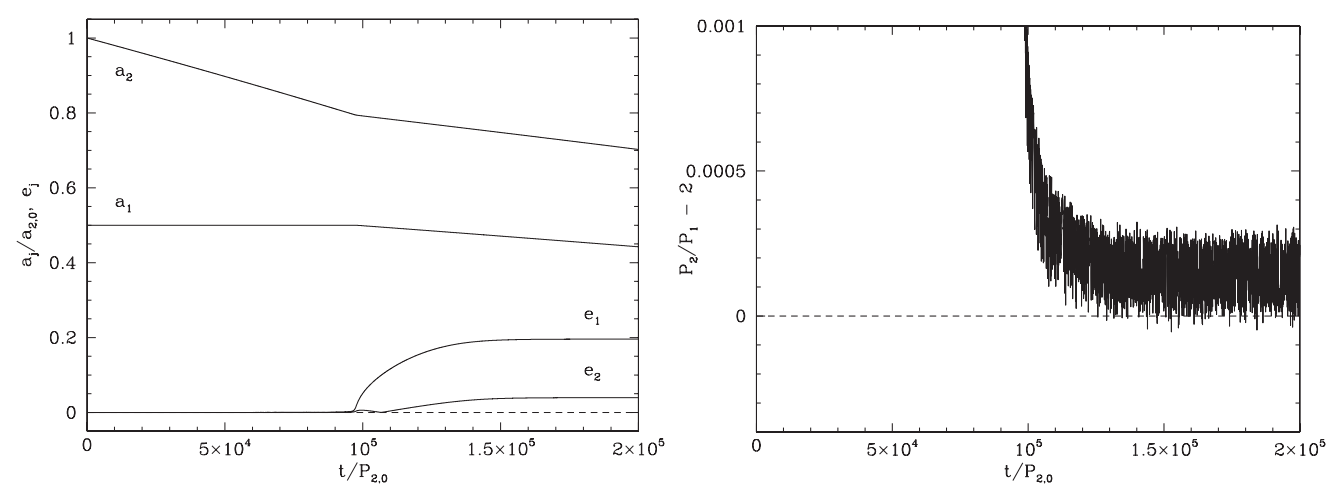

Figure 1. Evolution of $a_{i}, e_{i}$ and $P_{2} / P_{1}-2$ in a calculation where $M_{*}=1 M_{\odot}$, $M_{1}=M_{2}=10 M_{\oplus}$ and the outer planet is forced to migrate inward. The semimajor axes and time are in units of the initial $a_{2,0}$ and $P_{2,0}$ of the outer planet, respectively.

and the outer planet is forced to migrate inward with $\dot{a}_{2} / a_{2} \propto P_{2}^{-1}$ and $K_{e}=100$. The pair is captured into 2:1 resonance with both $\theta_{1}$ and $\theta_{2}$ librating. The eccentricities reach equilibrium values that are too large $\left(e_{1} \approx 0.2\right)$, and $P_{2} / P_{1}$ departs from 2 by less than 0.001 at the end. This result is representative of calculations with $K_{e} \sim 100$ for both 2:1 and 3:2. Hence the Kepler near-resonance pairs are not the result of eccentricity damping within the expected range during disk-induced migration.

\section{Tidal Damping of Eccentricity}

It has been proposed that the subsequent damping of orbital eccentricities by tidal dissipation in the planets may reduce the eccentricities to sufficiently small values to explain the observed departures from exact commensurabilities (Lithwick \& Wu 2012; Batygin \& Morbidelli 2012). Tidal dissipation in the planet damps the orbital eccentricity on timescale

$$
\tau_{e}=\frac{e}{\dot{e}}=\frac{1}{21 \pi} \frac{Q}{k_{2}} \frac{M_{p}}{M_{*}}\left(\frac{a}{R_{p}}\right)^{5} P,
$$

while conserving the total angular momentum of the system, where $R_{p}, Q$, and $k_{2}$ are the radius, tidal dissipation function, and Love number of the planet of mass $M_{p}$. The above equation assumes that the planet is synchronously rotating and that $Q$ is constant as a function of the tidal frequencies. Since $\dot{e} / e$ is independent of $e$ and $a$ changes only slightly for small $e$, one might expect $e$ to decay exponentially. However, for the planet pairs near a $j:(j-1)$ resonance, Lithwick \& Wu (2012), Batygin \& Morbidelli (2012), and Delisle et al. (2012) have shown analytically that the eccentricities decay slowly according to a shallow power law due to interactions between the planets:

$$
e_{1} \propto\left(t / \tau_{e}\right)^{-1 / 3}
$$

and

The coefficient

$$
\frac{P_{2}}{P_{1}}-\frac{j}{(j-1)}=\left(D_{j} t / \tau_{e}\right)^{1 / 3} .
$$

$$
D_{j}=\frac{9 j^{2}}{(j-1)^{3}}\left(\frac{M_{1}}{M_{*}}\right)^{2} \beta(1+\beta) C_{1}^{2},
$$



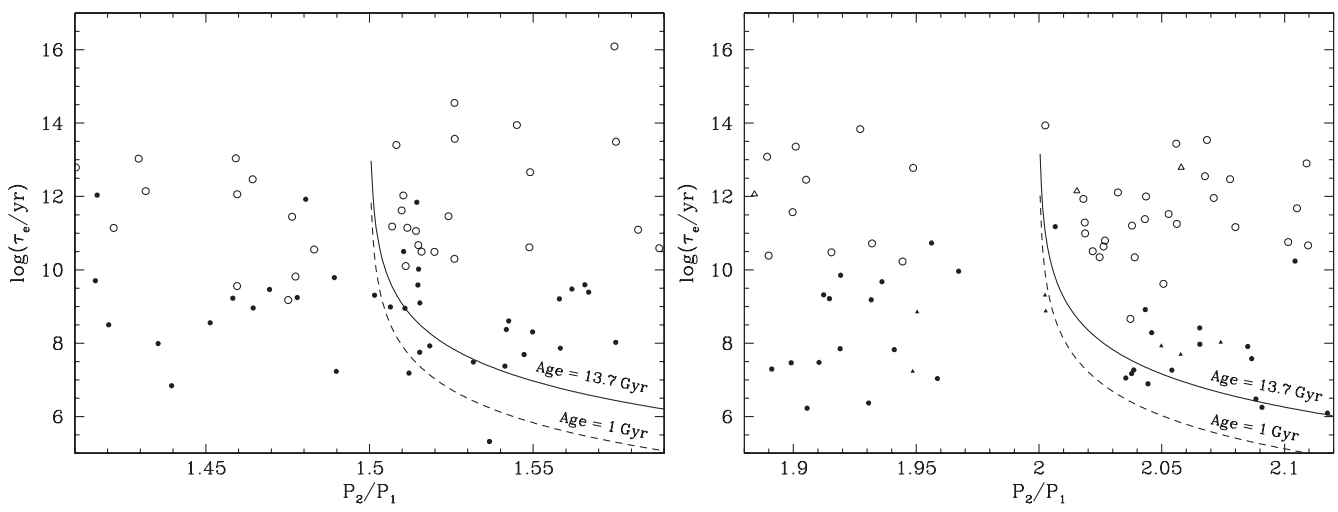

Figure 2. Tidal eccentricity damping timescale $\tau_{e}$ of the inner planet for the Kepler candidate pairs near the 3:2 and 2:1 resonances. See text for details.

where $\beta=\left(M_{2} / M_{1}\right) \alpha^{-1 / 2}=\left(M_{2} / M_{1}\right)[j /(j-1)]^{1 / 3}$, due to tidal dissipation in the inner planet only. (Tidal dissipation in the outer planet adds to $D_{j} / \tau_{e}$ but does not change the $1 / 3$ power-law behavior.) We have performed numerical simulations where the planets are initially in 2:1 or 3:2 resonance and the eccentricity of the inner planet is damped on a constant timescale $\tau_{e}$ (while the semimajor axis of the inner planet is adjusted at the same time to conserve orbital angular momentum) to simulate tidal dissipation in the inner planet. The numerical results are in excellent agreement with the above analytic result after an initial transient period of a few $\tau_{e}$.

An important consequence of this shallow power-law behavior is that many $\tau_{e}$ must elapse to produce a departure of $P_{2} / P_{1}$ of a few percent from exact commensurability. For example, $P_{2} / P_{1}-j /(j-1) \approx 0.03$ requires $t \gtrsim 50 \tau_{e}$. For $P=10$ days, $M_{*}=1 M$., $M_{p}=10 M_{\oplus}, R_{p}=3 R_{\oplus}, \tau_{e}=2.26 \times 10^{6}\left(Q / k_{2}\right)$ yr. Whether a near-resonance pair can reach its $P_{2} / P_{1}-j /(j-1)$ within the age of its host star ( $\sim$ a few Gyr) depends critically on $Q / k_{2}$ of the inner planet, which is very different for rocky and giant planets.

The tidal $Q / k_{2}$ of Solar System planets have been measured or constrained by the tidal evolution of their satellites, while those of extrasolar giant planets have been constrained by the existence of some close-in planets with non-zero orbital eccentricities. The lowest bound on $Q / k_{2}$ is 40 for rocky planets from Earth (Murray \& Dermott 1999) and $2.2 \times 10^{4}$ for giant planets from Neptune (Banfiled \& Murray 1992; Zhang \& Hamilton 2008).

\section{Comparison with Observations}

Figure 2 shows the tidal eccentricity damping timescale $\tau_{e}$ (eq. [3.1]) of the inner planet for the Kepler candidate pairs near the 2:1 and 3:2 resonances. Circles are adjacent pairs, and triangles are non-adjacent pairs. Filled and open symbols are pairs with the radius of the inner planet $R_{1}<2 R_{\oplus}$ and $R_{1}>2 R_{\oplus}$, respectively. For the "giant" planets with $R_{p}>2 R_{\oplus}$, we adopt $Q / k_{2}=10^{5}$ and mass from the mass-radius relationship $M_{p}=M_{\oplus}\left(R_{p} / R_{\oplus}\right)^{2.06}$ of Lissauer et al. (2011), which is consistent with Earth to Saturn in the Solar System and with the known extrasolar planets. For the "rocky" planets with $R_{p}<2 R_{\oplus}$, we adopt $Q / k_{2}=100$ and mass from the mass-radius relationship $M_{p}=M_{\oplus}\left(R_{p} / R_{\oplus}\right)^{3.7}$ of Valencia et al. (2006). The dashed and solid lines in Figure 2 show $\tau_{e}$ as a function of $P_{2} / P_{1}$ according to equations (3.3) and (3.4) for $t=1 \mathrm{Gyr}$ and $13.7 \mathrm{Gyr}$, respectively, if we have two $10 M_{\oplus}$ planets orbiting a solar-mass star (i.e., $M_{1} / M_{*}=3 \times 10^{-5}$ and $M_{1}=M_{2}$ ). These lines indicate where such a resonant pair 


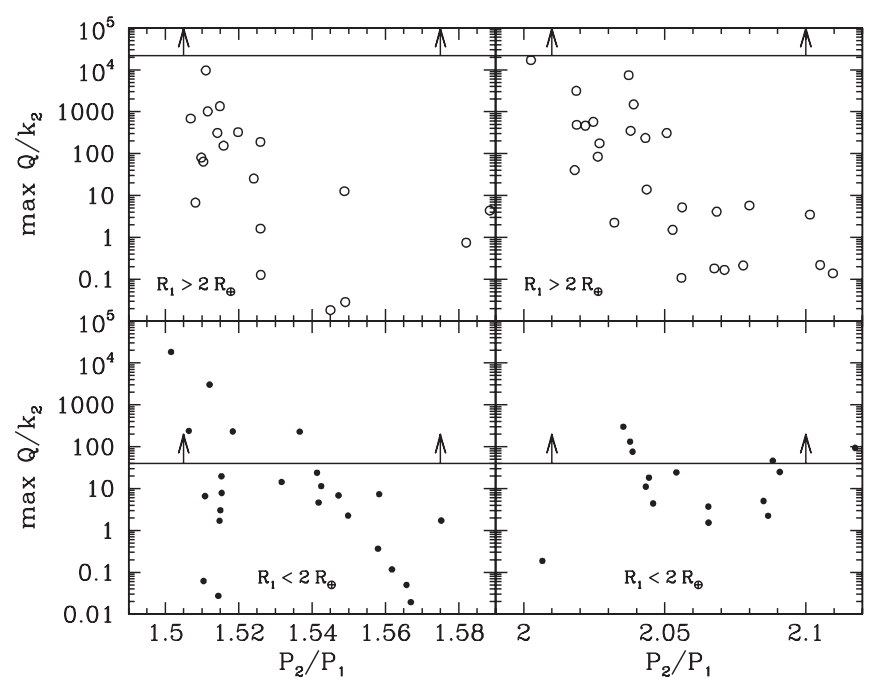

Figure 3. Maximum $Q / k_{2}$ for the inner planet of the candidate Kepler pairs, with the four panels showing the $R_{1}<2 R_{\oplus}$ and $R_{1}>2 R_{\oplus}$ cases for $3: 2$ and 2:1. See text for details.

would be in $P_{2} / P_{1}$, if it started near exact commensurability, its age is $t=1 \mathrm{Gyr}$ or 13.7 Gyr, and the tidal eccentricity damping timescale of the inner planet is $\tau_{e}$. There are some filled symbols below the solid lines for the age of the Universe, hinting that some of the near-resonance pairs with $R_{1}<2 R_{\oplus}$ can potentially reach their current locations by tidal eccentricity damping in less than the age of the host star. Most of the pairs with $R_{1}>2 R_{\oplus}$ (open symbols) are well above the lines and have $\tau_{e}>10^{10} \mathrm{yr}$, indicating that they are unlikely to reach their current locations by tidal eccentricity damping. However, the comparison in Figure 2 is not exact, as the theoretical curves are for a specific combination of stellar and planetary masses, and the observed Kepler pairs are plotted for assumed $Q / k_{2}$.

Instead of $\tau_{e}$, we can plot (eqs. [3.1] and [3.3])

$$
\frac{Q}{k_{2}}=\frac{21 \pi}{P_{1}} \frac{M_{*}}{M_{1}}\left(\frac{R_{1}}{a}\right)^{5}\left[P_{2} / P_{1}-j /(j-1)\right]^{-3} D_{j} t
$$

versus $P_{2} / P_{1}$ near the $j:(j-1)$ resonance with $t=13.7$ Gyr. This is the maximum $Q / k_{2}$ or minimum tidal dissipation efficiency that the inner planet must have if the pair is to evolve to its current $P_{2} / P_{1}$ in less than the age of the Universe. In Figure 3, the points show this maximum $Q / k_{2}$ for the inner planet of the observed Kepler pairs. The four panels show the $R_{1}<2 R_{\oplus}$ and $R_{1}>2 R_{\oplus}$ cases for $3: 2$ and $2: 1$. The lines with arrows pointing upward are the known lowest bound on $Q / k_{2}$ for planets $\left(2.2 \times 10^{4}\right.$ for giant planets and 40 for rocky planets). Figure 3 clearly shows that some pairs with $R_{1}<2 R_{\oplus}$ can reach where they are by tidal eccentricity damping, if planets with $R_{p}<2 R_{\oplus}$ are rocky with $Q / k_{2} \geqslant 40$, but that none of the pairs with $R_{1}>2 R_{\oplus}$ can reach their current $P_{2} / P_{1}$, if planets with $R_{p}>2 R_{\oplus}$ are giants with $Q / k_{2} \geqslant 2.2 \times 10^{4}$. Furthermore, there 
are clumps of $R_{1}>2 R_{\oplus}$ pairs just outside $2: 1$ and $3: 2$ that are more than an order of magnitude below $Q / k_{2}=2.2 \times 10^{4}$.

\section{Discussion}

We have shown that some of the Kepler near-resonance pairs with $R_{1}<2 R_{\oplus}$ may be able to move to their current near-resonance locations by tidal damping of eccentricity if they are rocky with $Q / k_{2} \sim 100$, but that planet pairs with $R_{1}>2 R_{\oplus}$ are unable to move their current near-resonance locations by the same mechanism if they are giants with $Q / k_{2} \gtrsim 2 \times 10^{4}$. What are the alternatives? One possibility is that some of the $R_{p}>2 R_{\oplus}$ planets are in fact rocky with low $Q / k_{2}$. Mass measurements or constraints would be a way to rule in or out this possibility. Otherwise, another mechanism is needed for the pairs with inner giant planets. Rein (2012) has suggested migration in turbulent disk, which has both smooth and stochastic components. The departure from exact commensurability can be used to constrain the relative strength of smooth and stochastic migration. However, Rein (2012) has assumed that the smooth migration is always inward, which is only true for classic type I (and type II) migration. Recent improvements in the analysis of the corotation and horseshoe torques (plus the differential Linblad torque) have shown that type I migration can be outward in some regions of certain disk models, and that there are locations in the disk where the total torque vanishes and the migration is stalled (e.g., Paardekooper et al. 2011; Kretke \& Lin 2012). This more complex migration behavior means that it is possible for a pair of planets to undergo both convergent and divergent migration, as the disk accretion rate decreases with time and the disk depletes. Whether the breaking of resonances by divergent migration could result in an excess of planet pairs just outside the first-order resonances will require further investigation.

\section{References}

Artymowicz, P. 1993, ApJ, 419, 166

Banfield, D. \& Murray, N. 1992, Icarus, 99, 390

Batygin, K. \& Morbidelli, A. 2012, AJ, 145, article id. 1

Bryden, G., Różyczka, M., Lin, D. N. C., \& Bodenheimer, P. 2000, ApJ, 540, 1091

Delisle, J.-B., Laskar, J., Correia, A. C. M., \& Boué, G. 2012, A\&A, 546, 71

Fabrycky, D. C., et al. 2012, ApJ, submitted (arXiv:1202.6328)

Kley, W. 2000, MNRAS, 313, L47

Kretke, K. A. \& Lin, D. N. C. 2012, ApJ, 755, 74.

Laughlin, G. \& Chambers, J. E. 2001, ApJ, 551, L109

Laughlin, G., Butler, R. P., Fischer, D. A., Marcy, G. W., Vogt, S. S., \& Wolf, A. S. 2005, ApJ, 622,1182

Lee, M. H. 2004, ApJ, 611, 517

Lee, M. H. \& Peale, S. J. 2002, ApJ, 567, 596

Lissauer, J. J., et al. 2011, ApJS, 197, 8

Lissauer, J. J., et al. 2012, ApJ, 750, 112

Lithwick, Y. \& Wu, Y. 2012, ApJ, 756, L11

Murray, C. D. \& Dermott, S. F. 1999, Solar System Dynamics (Cambridge: Cambridge Univ. Press)

Paardekooper, S.-J., Baruteau, C., \& Kley, W. 2011, MNRAS, 410, 293.

Rivera, E. J. \& Lissauer, J. J. 2001, ApJ, 558, 392

Rein, H. 2012, MNRAS, 427, L21

Tanaka, H., Takeuchi, T., \& Ward, W. R. 2002, ApJ, 565, 1257

Valencia, D., O'Connell, R. J., \& Sasselov, D. 2006, Icarus, 181, 545

Ward, W. R. 1997, Icarus, 126, 261

Zhang, K. \& Hamilton, D. P. 2008, Icarus, 193, 267 